\title{
Global Epidemic of Obesity and Diabetes: Where Did We Go Wrong?
}

\author{
Gundu HR Rao* \\ Laboratory Medicine and Pathology Director, USA \\ *Corresponding author: Gundu HR Rao, Laboratory Medicine and Pathology Emeritus Professor, Thrombosis Research, Lillehei Heart Institute, USA
}

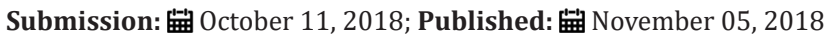

\section{Introduction}

According to the Harvard T. H. Chan School of Public Health, in 2012, more than a third of the US population was obese [1]. There is some speculation, that soon most adult population in the US, may become obese. Experts estimate, that obesity has increased in the last three decades, by two-fold and diabetes (type-2) during the same period, by four-fold worldwide. In 2016, more than 1.9 billion adults worldwide were overweight, and 650 million were obese, thus indicating the shift in the normal versus overweight population [2]. Surprisingly, the most advanced nation of the World, the USA is number one in the prevalence of highest diabetes population in the age between 27-79 years [3]. The 10 States with the highest type-2 diabetes rates are in the South. West Virginia has the highest adult rate diabetes at $15.2 \%$. Scientists at the Center for Disease Control (CDC) USA, have identified a diabetes belt. mostly in the Southern portion of the United States. The belt includes, Alabama, Arkansas, Florida, Georgia, Kentucky, Louisiana, North Carolina, Ohio, Pennsylvania, South Carolina, Tennessee, Texas, Virginia and West Virginia. Both obesity and diabetes are related, multifactorial, complex diseases and by and large are preventable. Having said, that one may wonder as to why, there is this rapid increase in these metabolic diseases worldwide, to "tsunami" levels? A study by Shen and associates in China, reports that diabetes has increased 17-fold in the last three decades [4]. They attribute this phenomenal rise in the incidence of diabetes, to social and economic development, urbanization, dietary pattern, and Westernized lifestyle. This is probably true, in many other developing countries around the globe. In 2012, the World Health Assembly (WHA), set a global target to reduce deaths from non-infectious disease- including cardiovascular disease-by 25 percent by 2025 . No country has reduced, reversed, or halted the increase in the incidence of obesity and diabetes.

Considering the rates of severe obesity in children in the USA, in 2010, the Whitehouse established the first ever Task Force, on childhood Obesity to end this public health problem within one generation. Follow-up reports indicate, significant progress in communities across the nation. Obesity is a major risk factor, for the development of type-2 diabetes. Added to this observation, it is worthwhile considering another contributing factor, pre-diabetes. According to a NHANES 2011-2012 survey, prevalence of predia betes was $36.5 \%$ among US adults, which is three-fold greater than diabetes incidence in the USA. Obesity and diabetes contribute significantly, to the development of vascular pathology. They produce a variety of structural and functional alterations. Like the obesity and diabetes, CVD burden varies among the US states [5]. The highest rate of CVD burden was in Mississippi, followed by Arkansas, Oklahoma, Louisiana, Alabama, Kentucky, West Virginian, South Carolina, and Georgia. The lowest CVD burden was in Minnesota, followed by New England and Pacific Northwest, including Massachusetts, New Hampshire, Washington, Connecticut, Vermont and Oregon. It took more than 25 years for the highest burden states to catch up, with the states with the lowest CVD burden. Just think how long will it take for various sociodemographic regions in the world, to catch up with the progress made in industrialized nations, and what would be the burden of these chronic diseases by that time worldwide?

What choice do we have to halt, reduce, reverse, or prevent these epidemics of obesity and diabetes worldwide? American Diabetes Association says, over 30 million Americans live with diabetes; 84 million more have prediabetes and the epidemic keeps growing. Unless we act now, one in three Americans will be diagnosed with this disease by 2050 . Diabetes was identified as one of four priority non-communicable diseases, targeted by the United Nation due to its growing disease burden. The Sustainable Development Goals also include, a target to reduce the proportion of premature deaths due to NCDs, including diabetes, by one third by 2030. Global Burden of Disease Study 2010 (GBD2010), a collaborative project led by the Institute for Health Metrics and Evaluation (IHME), at the University of Washington, announced its findings to the Royal Society London, and published the results of these studies in the Lancet for the first time, an entire triple issue devoted to the findings of this one study. Robust management of modifiable risk factors for CVD development, have reduced significantly CVD-related deaths in industrialized nations. Following a healthy diet with increased physical activity, seems to be the most effective, least expensive choice for reducing, or reversing this disease burden.

Compliance is a major problem worldwide. For instance, in the USA, it is estimated that over a million are not taking any aspirin 
as recommended. Fifty million, use tobacco or tobacco products. Seventy million are physically inactivate. In countries like China and India, with large populations, millions of individuals with metabolic disease are not under any preventive protocols. Dr Hiroyuki Sagesaka and associates from Aizawa Hospital in Matsumoto, Japan, have identified that just here risk factors, increase in fasting glucose, BMI, and insulin resistance, can be used to predict future prediabetes 10 years before it occurs. They conclude that clear majority of people with type- 2 diabetes go through the stage of prediabetes, and these findings suggest that elevated metabolic markers for diabetes are detectable more than 20 years before its diagnosis. These findings were presented at this year's European Association for the Study of Diabetes (EASD), Annual Meetings in Berlin, Germany (October 2018). Despite these findings, screening healthy individuals in countries with large populations, like China and India, poses a great logistical, infrastructural, and economic challenge. To date, the best evidence for prevention of obesity and diabetes epidemic, come from randomized trials of lifestyle interventions. The purpose of this editorial is to discuss some of the problems related to this disease epidemic, identify the problem areas, articulate the need for further improvement in awareness programs, educational programs, and existing preventive strategies. I sincerely urge the readers, to contemplate on these global public health problems, respond with positive comments and suggestions.

\section{References}

1. Bhupathiraju SN, Hu FB (2016) Epidemiology of obesity and diabetes and their cardiovascular complications. Circ Res 118 (11):1723-1735.

2. Nyberg ST, Batty GD, Pentti J, Virtanen M, Alfredsson L, et al. (2018) Obesity and loss of disease-free years owing to major non-communicable diseases: a multicohort study. Lancet Public Health 3(10): 490-497.

3. Endocrine News: US Leads Developed Nations in Diabetes Prevalence, USA.

4. Shen X, Vaidya A, Wu S, Gao X (2016) The diabetic epidemic in China: An integrated review of national surveys. Endocr Pract 22(9): 1119-1129.

5. Global Burden of the Cardiovascular Disease Collaboration: The burden of cardiovascular diseases among the US States, 1990-2016.
Creative Commons Attribution 4.0 International License

For possible submissions Click Here

\section{Submit Article}

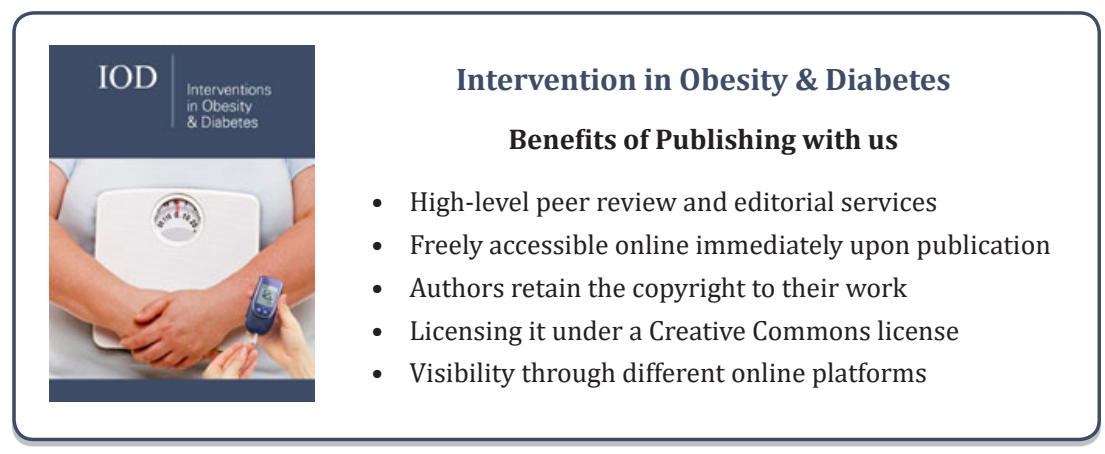

